\title{
The Imbalance Between Metalloproteinases and Their Tissue Inhibitors Is Involved in the Pathogenesis of Dermatitis Herpetiformis
}

\author{
Agnieszka Zebrowska, ${ }^{1}$ Joanna Narbutt, ${ }^{1}$ Anna Sysa-Jedrzejowska, ${ }^{1}$ Jozef Kobos, ${ }^{2}$ and Elzbieta Waszczykowska ${ }^{3}$ \\ ${ }^{1}$ Department of Dermatology and Venereology, Medical University of Lodz, 5 Krzemieniecka Street, 94017 Lodz, Poland \\ ${ }^{2}$ Laboratory of Immunopathology, Department of Children Diseases, Medical University of Lodz, \\ 5 Krzemieniecka Street, 94017 Lodz, Poland \\ ${ }^{3}$ Laboratory of Immunodermatology, Department of Dermatology and Venereology, \\ Medical University of Lodz, 5 Krzemieniecka Street, 94017 Lodz, Poland
}

Received 1 June 2005; accepted 8 August 2005

\begin{abstract}
Dermatitis herpetiformis (DH) is a subepidermal autoimmune disease characterized by skin and intestinal lesions consistent with coeliac disease. There are also some data that metalloproteinases (MMPs) are involved in the development of skin lesions in DH, however their exact role in this process is not fully understood. The aim of the study was to investigate whether MMPs and their inhibitors are involved in pathogenesis of DH. Skin biopsies were taken from 13 patients with active DH and from 10 healthy subjects. The localization and expression of MMPs and TIMPs were examined by immunohistochemistry. MMPs expression was detected in basal keratinocytes and in the whole epidermis in all of the DH subjects. Neutrophils in microabscesses and in blister fluid were also positive for MMPs. Expression of TIMPs was moderate or weak in all examined biopsies. Our results allow us to conclude that imbalance between these enzymes takes an important role in the pathogenesis of DH.
\end{abstract}

\section{INTRODUCTION}

Dermatitis herpetiformis $(\mathrm{DH})$ is one of the subepidermal autoimmune diseases characterized by skin and intestinal lesions. Skin lesions include polymorphic eruption (papules, vesicles) accompanied by severe pruritus. Intestinal lesions, consistent with coeliac disease, are characterized by atrophy of intestinal villi resulting in immunological process. In most cases intestinal disturbances are subclinical, however a few patients have been presented with diarrhoea, malabsorption, and loss of body weight. Diagnosis of $\mathrm{DH}$ is established on the base of the results of direct immunofluorescent test (granular deposits of IgA in the papillae) and the presence of circulating IgA antibodies directed against endomysium and/or tissue transglutaminase. Skin lesions in DH are histologically characterized by neutrophilic inflammatory infiltrate leading to destruction of basement membrane zone proteins. Impairment of type IV collagen, laminin, and entactin results in degradation of anchoring fibers and blister formation [1].

Correspondence and reprint requests to Agnieszka Zebrowska, Department of Dermatology and Venereology, Medical University of Lodz, 5 Krzemieniecka Street, 94017 Lodz, Poland; zebrowskaaga@wp.pl
Recent data sugested that the main autoantigen in $\mathrm{DH}$ is tissue transglutaminase. This enzyme has two isoforms: tissue and epidermal [2, 3]. Binding of circulating antitissue transglutaminase $\operatorname{IgA}$ antibodies with the antigen causes formation of deposits in skin papillae and activation of complement followed by influx of neutrophiles and release of proinflammatory cytokines $[4,5]$. Various inflammatory agents, including cytokines, are involved in many biological processes, among others in overproduction of metalloproteinases, especially stromelysins, gelatinases, and matrilysins [6].

Matrix metalloproteinases (MMPs) and their inhibitors (tissue inhibitors of matrix proteinases, TIMPs) are known to play a significant role in a number of physiological and pathological processes. MMPs are a family of zinc-dependent endopeptidases and their main function is the degradation of all components of extracellular matrix. Balance between MMPs and TIMPs is required for morphogenesis, homeostasis, and tissue repair. An increased expression of MMPs and a decreased expression of TIMPs may occur in cancer, artheriosclerosis, and connective tissue diseases [7].

Although metalloproteinases might be involved in the development of skin lesions in $\mathrm{DH}$, their precise role in this process is not fully understood [8]. The goal of the study was to investigate matrix metalloproteinases 
and their inhibitors expression and involvement in the pathogenesis of DH. Collagenase (MMP1), gelatinase (MMP2), $92 \mathrm{kd}$ gelatinase (MMP9), and stromelysin 2 (MMP10) and TIMP1, TIMP2, TIMP3 tissue expression and localization were examined by immunohistochemistry in lesional skin biopsies. Understanding the role of these enzymes in the mechanisms of skin lesion formation may contribute to the development of new therapeutic strategies.

\section{MATERIAL AND METHODS}

\section{Patients}

Thirteen patients ( 5 women, 8 men, mean age 44.78 years, range 18 to 64 ) in active stage of $\mathrm{DH}$ entered the study. Seven out of 13 patients had skin lesions characterized by vesicles and itching papules, whereas others had only erythematous papules.

Control group consisted of 10 patients ( 5 women and 5 men, mean age 42 years, range 19-49), recruited from volunteers. Control group has not skin lesions.

DH diagnosis was established based on history, clinical and histological features, and immunological tests [9]. Before entering the study all the patients and controls gave written informed consent and the study was approved by the local Ethics Committee of Medical University of Lodz.

Skin pathology. In all study, subject histological examination showed neutrophilic infiltrates, the presence of Pierard's abscesses, and-in some cases-subepidermal blisters. In 7 samples, unilocular blisters displaying multiple neutrophilic papillary microabscesses were found. Typical papillary microabscesses and very small vesicles were observed in 4 biopsies.

Immunological characteristics of the patients. Direct immunofluorescence test revealed the presence of granular deposits of IgA in skin papillae. Indirect immunofluorescence test was positive for IgAEmA (Oesophagus monkey IgAEmA, Medizinische Labordiagnostica, Denmark), in all the patients (titer 1:10-1:320, median 1:40). Anti-tissue transglutaminase antibodies measured using ELISA (Celikey, Pharmacia \& Upjohn Freiburg, Germany) method were present in 4 out of 13 cases (median $4.3 \mathrm{JU} / \mathrm{mL})$.

\section{Tissues}

Formalin-fixed, paraffin-embedded biopsy specimens $(n=13)$ lesions from buttock skin were taken. Additionally biopsy specimens were taken from buttock skin from 10 healthy volunteers, age- and sex-matched with patients.

Immunohistochemistry. Paraffin-embedded sections (3-4 $\mu \mathrm{m}$ thick) were used for routine $\mathrm{H}+\mathrm{E}$ staining and for immunohistochemistry in DAKO EnVision detection system using immunoperoxidase method. The following primary mouse monoclonal antibodies were used: antiMMP1, anti-MMP2, anti-MMP9, anti-MMP10 and antiTIMP1, anti-TIMP2, and anti-TIMP3 (Novocastra).
For immunohistochemistry, the paraffin-embedded sections were placed on adhesive plates and dried at $56^{\circ} \mathrm{C}$ for 24 hours, later they were deparaffinated in a series of xylen and alcohol with decreasing concentrations $(96 \%, 80 \%, 70 \%, 60 \%)$. Activity of endogenous peroxidase was inhibited with 3\% hydrogen peroxide solution in methanol for 5 minutes.

In order to retrieve the antigenicity of tissues and allow them to react with antibodies, specific procedures were used for each antibody, according to manufacturers' instruction. After incubation with diluted antibodies for 60 minutes at room temperature, slidos were washed with TRIS buffer twice. Then, DAKO EnVision doublestep visualization system was applied to visualize the antigen-antibody reaction. In cases of positive immunohistochemical reaction, cellular nuclei were stained with Meyer's haematoxylin for 2 minutes. After dehydratation and processing through series of acetone and xylene as described above, the sections were fixed in Canadian balm.

The three-step semiquantitative scale was applied in the evaluation of the intensity of immunohistochemical reaction of markers used. The first step (weak intensity): immunohistochemical reaction was limited to the single epithelial cells and/or was only focally present in the stroma. The second step (moderate intensity): immunohistochemical reaction was observed in part of epithelial cells and/or was present in some areas of the stroma. The third step (strong intensity): immunohistochemical reaction was observed in numerous epithelial cells and/or was present in large areas of the stroma.

Expression of four MMPs and TIMPs was assessed by two independent pathologists using Nikon Microfob FXA microscope (Nikon LTD, Japan).

\section{RESULTS}

\section{Immunohistochemistry}

Detailed characteristic of staining is presented in Table 1.

\section{Collagenase (MMP1)}

A signal for collagenase was detected in basal keratinocytes in all of the samples; in 8 out of 13 it was very intensive. Collagenase expression was not only limited to basal keratinocytes but it was also detected in the whole epidermis and its intensity was moderate (Figure 1). Polymorphonuclear lymphocytes in microabscesses (4 out of 13) and in blister fluid (5 out of 7) showed positive staining as well. Signal for MMP1 was detected also in infiltrates of neutrophiles located around microabscesses and in perivascular infiltrates. A moderate expression for MMP1 was also found in stromal cells in 5 out of 13 biopsies.

\section{Gelatinase (MMP2)}

In skin samples (13 out of 13), high expression of MMP2 was found in basal keratinocytes, while in other 
TABLE 1. Expression of MMPs and TIMPs in examined tissues. ND: nondetectable.

\begin{tabular}{|c|c|c|c|c|c|c|c|}
\hline \multicolumn{8}{|c|}{ Localization } \\
\hline \multicolumn{8}{|c|}{ Positive biopsies/all biopsies } \\
\hline Enzyme & $\begin{array}{c}\text { Basal } \\
\text { keratinocytes }\end{array}$ & $\begin{array}{l}\text { Whole } \\
\text { epidermis }\end{array}$ & Microabscesses & Infiltration & $\begin{array}{l}\text { Stromal } \\
\text { cells }\end{array}$ & $\begin{array}{l}\text { Hair } \\
\text { follicle }\end{array}$ & $\begin{array}{c}\text { Blister } \\
\text { fluid }\end{array}$ \\
\hline Patients & & & $(N=13)$ & & & & $(N=7)$ \\
\hline MMP1 & $13 / 13$ & $13 / 13$ & $4 / 13$ & $13 / 13$ & $5 / 13$ & $\mathrm{ND}$ & $5 / 7$ \\
\hline MMP2 & $13 / 13$ & $13 / 13$ & $4 / 13$ & $1 / 13$ & $3 / 13$ & ND & $5 / 7$ \\
\hline MMP9 & $13 / 13$ & $9 / 13$ & $7 / 13$ & $2 / 13$ & $3 / 13$ & ND & $6 / 7$ \\
\hline MMP10 & $13 / 13$ & $12 / 13$ & $7 / 13$ & $9 / 13$ & $2 / 13$ & ND & $5 / 7$ \\
\hline TIMP1 & $13 / 13$ & $13 / 13$ & $9 / 13$ & $5 / 13$ & $2 / 13$ & ND & $7 / 7$ \\
\hline TIMP2 & $13 / 13$ & $13 / 13$ & $7 / 13$ & $4 / 13$ & $3 / 13$ & ND & $4 / 7$ \\
\hline TIMP3 & $13 / 13$ & $13 / 13$ & $6 / 13$ & $4 / 13$ & $3 / 13$ & ND & $3 / 7$ \\
\hline Controls & \multicolumn{7}{|c|}{$(N=10)$} \\
\hline MMP1 & $\begin{array}{c}\text { Single } \\
\text { keratinocytes }\end{array}$ & - & - & - & - & $4 / 10$ & - \\
\hline MMP2 & $\begin{array}{c}\text { Single } \\
\text { keratinocytes }\end{array}$ & - & - & - & - & $4 / 10$ & - \\
\hline MMP9 & $\begin{array}{c}\text { Single } \\
\text { keratinocytes }\end{array}$ & - & 一 & - & - & $4 / 10$ & - \\
\hline MMP10 & $\begin{array}{c}\text { Single } \\
\text { keratinocytes }\end{array}$ & - & - & - & - & $4 / 10$ & - \\
\hline TIMP1 & $\begin{array}{c}\text { Single } \\
\text { keratinocytes }\end{array}$ & - & - & - & - & $4 / 10$ & - \\
\hline TIMP2 & $\begin{array}{c}\text { Single } \\
\text { keratinocytes }\end{array}$ & - & - & - & - & $4 / 10$ & - \\
\hline TIMP3 & $\begin{array}{c}\text { Single } \\
\text { keratinocytes }\end{array}$ & - & - & - & - & $4 / 10$ & - \\
\hline
\end{tabular}

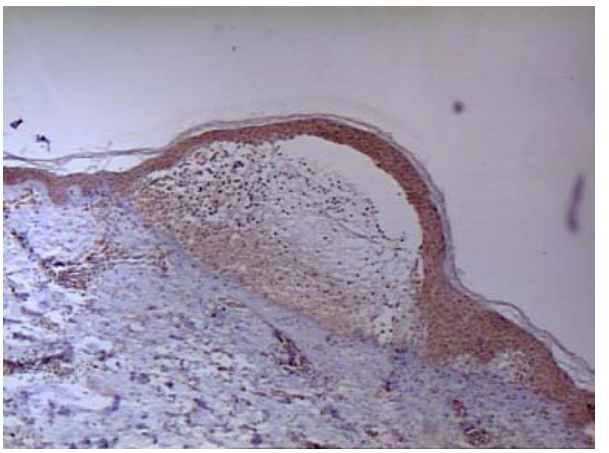

FIGURE 1. Skin lesions. Immunohistochemistry. Moderate expression of MMP1 in the whole epidermis and in blister fluid (magnification: $\times 100$ ).

layers of the epidermis MMPs expression was moderate. Positive staining was also observed in polymorphonuclear lymphocytes in microabscesses (4 out of 13) and in blister fluid ( 5 out of 7). Only in one biopsy, MMP2 expression was detected in infiltrates of neutrophiles located around microabscesses and in perivascular infiltrates. In 3 out of 13 biopsies a moderate expression of MMP2 in stromal cells was also found.

\section{2 kd gelatinase (MMP9)}

High expression of MMP9 was found in 3 out of 13 specimens in basal keratinocytes and in other biopsies the expression of that metaloproteinase in basal keratinocyte was moderate. In 9 out of 13 biopsies, its expression was observed in the whole epidermis. Polymorphonuclear lymphocytes in microabscesses (7 out of 13) and in blister fluid (6 out of 7 ) revealed positive staining as well. In 2 biopsies, expression of MMP9 was detected in infiltrates of neutrophiles located around microabscesses and in perivascular infiltrates. A moderate signal for MMP9 was also found in stromal cells in 3 out of 13 biopsies.

\section{Stromelysin 2 (MMP10)}

In 4 out of 13 biopsies, the expression of MMP10 in basal kerationocytes was high (Figure 2) and in the rest 9 moderate. In 12 biopsies the moderate expression was observed in the whole epidermis. Polymorphonuclear lymphocytes in microabscesses (7 out of 13) and in blister fluid (5 out of 7) showed positive staining as well. In 9 biopsies a signal for MMP10 was detected in infiltrates of neutrophiles located around microabscesses and in perivascular infiltrates. A moderate signal for MMP10 was also found in stromal cells in 2 out of 13 biopsies. 


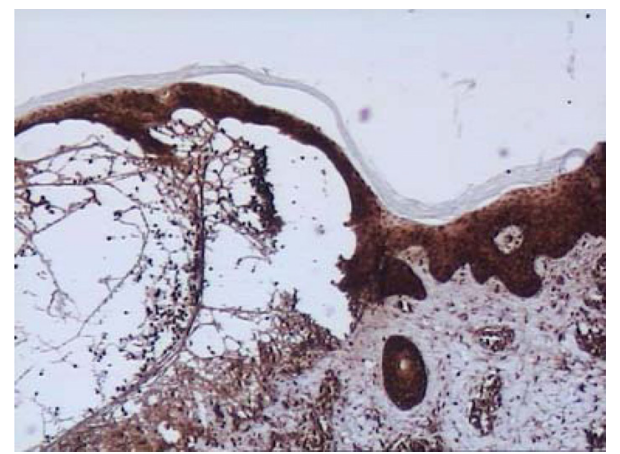

Figure 2. Skin lesions. Immunohistochemistry. High expression of MMP10 in the whole epidermis and in blister fluid (magnification: $\times 100$ ).

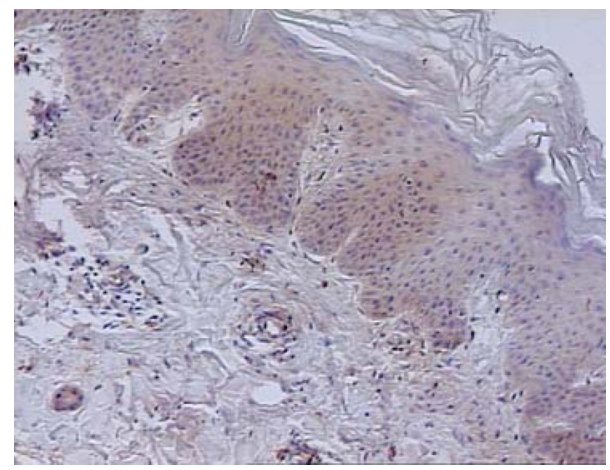

Figure 3. Skin lesions. Immunohistochemistry. Weak expression of TIMP1 in the whole epidermis (magnification: $\times 100$ ).

\section{TIMP1}

In contrast to metaloproteinases, expression of TIMPs was assessed as moderate or weak in all examined biopsies (Figure 3).

Moderate expression of TIMP1 was found in the whole epidermis including basal keratinocytes in all biopsies as well as in 9 out of 13 specimens in polymorphonuclear lymphocytes in microabscesses. It was also revealed in all ( 7 out of 7) biopsies in blister fluid. In 5 biopsies, a signal for TIMP1 was detected in infiltrates of neutrophiles located around microabscesses and in perivascular infiltrates.

\section{TIMP2}

Moderate expression of TIMP2 was found in the whole epidermis including basal keratinocytes in all biopsies (13 out of 13). Polymorphonuclear lymphocytes in microabscesses ( 7 out of 13) and in blister fluid ( 4 out of 7) also showed positive staining. In 4 biopsies, expression of TIMP2 was detected in infiltrates of neutrophiles located around microabscesses and in perivascular infiltrates. Moderate expression of TIMP2 was also found in stromal cells in 3 out of 13 biopsies.

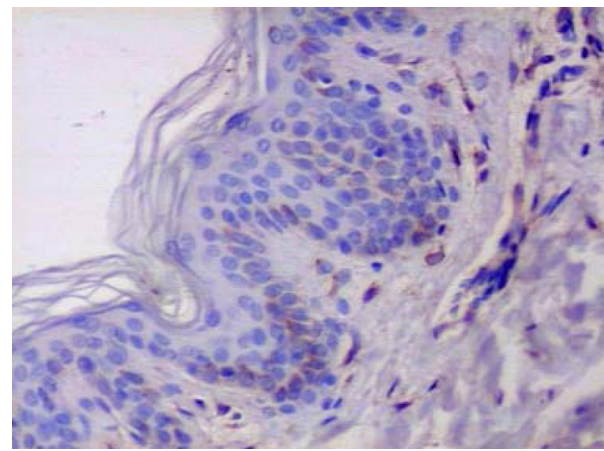

FIGURE 4. Healthy skin. Immunohistochemistry. Expression of MMP1 in single basal keratinocyte. Biopsy from healthy individual (magnification: $\times 400$ ).

\section{TIMP3}

Weak expression of TIMP3 was found in the whole epidermis including basal keratinocytes in all biopsies (13 out of 13). Polymorphonuclear lymphocytes in microabscesses (6 out of 13) and in blister fluid (3 out of 7) showed positive staining; however it was significantly weaker when compared to TIMP1 or TIMP2. In 4 biopsies, expression of TIMP3 was detected in neutrophil infiltrates located around microabscesses and in perivascular infiltrates and in 3 specimens in stromal area.

\section{Controls}

Expression of MMP1, MMP2, MMP9, MMP10 and TIMP1, TIMP2, TIMP3 was examined using the same method in 10 skin samples obtained from healthy volunteers. In all biopsies, only few basal keratinocytes showed moderate expression of all the proteins (Figures 4 and 5). In 4 hair-follicle biopsies, positive weak staining for the above enzymes was present.

\section{DISCUSSION}

Both soluble and membrane-anchored metaloproteinases participate in degradation of extracellular matrix (ECM) and might be involved in the development of skin lesions which are present in various subepidermal bullous diseases such as dermatitis herpetiformis, pemphigoid, and pemphigus $[7,10,11]$.

Although pathogenesis of DH is still not fully elucidated, there is an increasing evidence for primary role of tissue transglutaminase. Recent studies suggested that antibodies deposits formation in skin papillae directed against tissue transglutaminase activates complement and leads to accumulation of neutrophils with subsequent inflammation and degradation of extracellular components $[12,13]$. These phenomena are followed by formation of characteristic histological and clinical features, that is, subepidermal bullae and vesicles. It has been postulated that formation of blisters might be a result of local metalloproteinases $[12,13]$. 


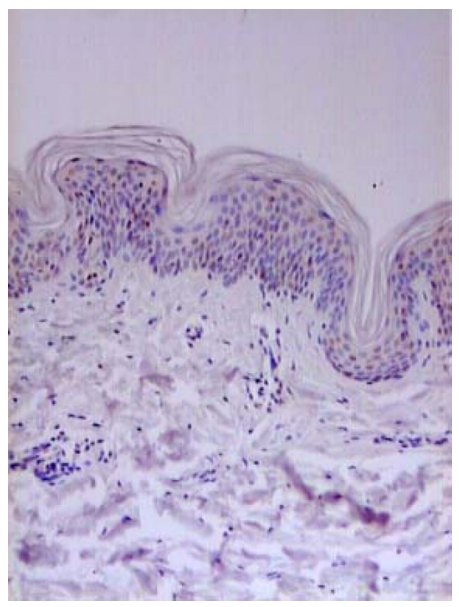

FIGURE 5. Healthy skin. Immunohistochemistry. Expression of TIMP1 in single keratinocyte (magnification: $\times 400$ ).

Moreover, the neutrophiles present in microabscesses release IL- $1 \beta$ stimulating keratinocytes and fibroblasts to produce collagenase [14]. On the other hand, experimental studies revealed that transglutaminase crosslinking to anchoring fibres in the epidermis may also significantly contributes to impairment of dermo-epidermal junction [15].

There are some data on the localization and intensity of expression of various MMPs in skin lesions obtained from $\mathrm{DH}$ patients.

Airola et al [16] found that interstitial collagenase is expressed only by basal keratinocytes in experimentally induced DH lesions. According to our results, although MMP1, MMP2, MMP9, and MMP10 expression is not restricted only to basal keratinocytes, the strongest expression was detected just in this layer of the epidermis. Increased amounts of matrix metalloproteinases have been demonstrated also in blister fluid. Several authors $[10,17]$ revealed that collagenase and elastase released by polymorphonuclear neutrophiles, in addition to keratinocyte metaloproteinases, are involved in the development of DH skin lesions. It is possible that neutrophiles might be the main source of collagenase activity in DH lesions $[11,18]$.

We have shown the presence of MMP1 in whole epidermis and in infiltrates of neutrophils and microabscesses in all (13/13) biopsies; however in some cases it was also present in blister fluid and stromal cells (5/13, 3/13 resp).

Very similar results refer to MMP2 (see Table 1); however its expression in neutrophils and in perivascular infiltrates was observed only in one specimen. We have shown that MMP2, similarly to MMP1, was highly expressed in all keratinocytes. It was also observed in microabscesses, blister fluid, and stromal cells (similarly to MMP1). Many authors showed that MMP2 may originate from multiple types of cells, mainly from dermal fibroblasts [19], but also from macrophages [20], keratinocytes [21], T lymphocytes [22], endothelial cells [23], and vascular smooth muscle cells [24]. Therefore it is possible that large amount of neutrophils in $\mathrm{DH}$ lesions was the reason for weaker expression of MMP2 in our samples.

Gelatinase (MMP2) is a potent degradating enzyme of the basement membrane. Most studies [25], including our results, showed its expression in the whole epidermis. The localization of MMP2 signal correlates with collagenase and stromelysine signals.

A strong expression of MMP9 and MMP10 was seen in basal keratinocytes only in 3 and 4 (resp) out of 13 biopsies. In almost all specimens a moderate expression for those proteins was detected in upper layers of keratinocytes and in case of MMP10 in infiltrates of neutrophils around microabscesses and in perivascular infiltrates. A moderate expression of MMP9 and MMP10 was detected in microabscesses $(7 / 13,7 / 13$, resp) and blister fluid $(6 / 13,5 / 13$, resp). In stromal cells, only in $2 / 13$ biopsies moderate expression for MMP9 and MMP10 was found.

MMP9 and MMP10 probably contribute to the formation of DH blisters by degrading basement membrane components [26]. We are in favour of this suggestion as we observed their high expression in basal keratinocytes and areas of intensive accumulation of neutrophils. Our results may suggest that not only basal keratinocytes are able to stimulate production of metalloproteinases.

Some authors $[8,16]$ found that overexpression of matrix metalloproteinases may be due to the release of cytokines by neutrophiles or by disregulation of plasmin system.

In previous studies [27] it was demonstrated that blister fluid obtained from various bullous dermatoses contains active proteases able to degrade collagen and elastin. Particularly high levels of metalloproteinases (collagenase, elastase) expression were present in $\mathrm{DH}$ blister fluids $[27,28]$. Increased collagenase activity in blister fluid was detected by Oikarinen et al [6], which is consistent with our results.

In this study we observed high expression of all examined MMPs in blister fluids. So we suppose that blister fluid may be a good material for the assessment of concentration of MMPs in skin lesions.

One of the hypothesis on DH lesion development involves the stimulation of collagenase inhibitor synthesis by dermal fibroblasts. Interleukin-1 and other cytokines released by epidermal and migrating cells under inflammatory process stimulate fibroblasts to produce both collagenase [29] and its inhibitor [30]. Disregulation between these two enzyme families subsequently may result in blister formation [31]. TIMPs block the activity of a number of matrix metalloproteinases $[32,33]$. That is why it is possible that their low expression might play a role in the development of subepidermal blistering diseases.

Intensity of expression of TIMP1, TIMP2, and TIMP3 in DH lesional biopsies was significantly lower as compare to MMPs; however expression of these enzymes was detected in all keratinocytes as well as in most of microabscesses and blister fluids. Less often, it was observed 
in infiltrates of neutrophiles around microabscesses, in perivascular infiltrates, and in stromal cells.

Based on our results we may suggest that TIMPs' expression in active DH lesions is significantly lower than MMPs. It is not clear whether overexpression of MMPs or low expression of TIMPs plays more important role in $\mathrm{DH}$ skin lesion formation. Our results allow us to conclude that imbalance between these groups of enzymes occurs and it may play an important role in the pathogenesis of the disease.

According to Liu et al [34] we also hope that therapeutic methods targeted on this subject might be an option to commonly used treatment.

More studies focused on this topic are needed to elucidate the role of MMPs and TIMPs in DH pathogenesis.

\section{ACKNOWLEDGMENTS}

This study was funded by the research projects of Medical University of Lodz, Poland: 503-119-1 and 50211-089. We would like to thank Ms Grazyna Jastrzebska and Stanislawa Madalinska for technical support.

\section{REFERENCES}

[1] Reunala TL. Dermatitis herpetiformis. Clin Dermatol. 2001;19(6):728-736.

[2] Dieterich W, Ehnis T, Bauer M, et al. Identification of tissue transglutaminase as the autoantigen of celiac disease. Nat Med. 1997;3(7):797-801.

[3] Sardy M, Karpati S, Merkl B, Paulsson M, Smyth N. Epidermal transglutaminase (TGase 3) is the autoantigen of dermatitis herpetiformis. J Exp Med. 2002;195(6):747-757.

[4] Zone JJ, Meyer L, Petersen M. Deposition of granular IgA relative to clinical lesions in dermatitis herpetiformis. Br J Dermatol. 1994;131:822-826.

[5] Caproni M, Feliciani C, Fuligni A et al. Th2-like cytokine activity in dermatitis herpetiformis. $\mathrm{Br} J \mathrm{Der}$ matol. 1998;138(2):242-247.

[6] Oikarinen AI, Reunala T, Zone JJ, Kiistala U, Uitto J. Proteolytic enzymes in blister fluids from patients with dermatitis herpetiformis. Br J Dermatol. 1986;114(3):295-302.

[7] Nagase H, Woessner JF Jr. Matrix metalloproteinases. J Biol Chem. 1999;274:21491-21494.

[8] Airola K, Vaalamo M, Reunala T, Saarialho-Kere UK. Enhanced expression of interstitial collagenase, stromelysin-1, and urokinase plasminogen activator in lesions of dermatitis herpetiformis. J Invest Dermatol. 1995;105(2):184-189.

[9] Chorzelski TP, Beutner EH, Jablonska S, Blaszczyk $\mathrm{M}$, Triftshauser C. Immunofluorescence studies in the diagnosis of dermatitis herpetiformis and its differentiation from bullous pemphigoid. J Invest Dermatol. 1971;56(5):373-380.
[10] Welgus HG, Bauer EA, Stricklin GP. Elevated levels of human collagenase inhibitor in blister fluids of diverse etiology. J Invest Dermatol. 1986;87(5):592596.

[11] Oikarinen AI, Zone JJ, Ahmed AR, Kiistala U, Uitto J. Demonstration of collagenase and elastase activities in the blister fluids from bullous skin diseases. Comparison between dermatitis herpetiformis and bullous pemphigoid. J Invest Dermatol. 1983;81(3):261-266.

[12] Dahl MV, Falk RJ, Carpenter R, Michael AF. Membrane attack complex of complement in dermatitis herpetiformis. Arch Dermatol. 1985;121(1):70-72.

[13] Graeber M, Baker BS, Garioch JJ, Valdimarsson H, Leonard JN, Fry L. The role of cytokines in the generation of skin lesions in dermatitis herpetiformis. Br J Dermatol. 1993;129(5):530-532.

[14] Petersen MJ, Woodley DT, Stricklin GP, O'Keefe EJ. Production of procollagenase by cultured human keratinocytes. J Biol Chem. 1987;262(2):835-840.

[15] Aeschlimann D, Paulsson M. Cross-linking of laminin-nidogen complexes by tissue transglutaminase. A novel mechanism for basement membrane stabilization. J Biol Chem. 1991;266(23):1530815317.

[16] Airola K, Reunala T, Salo S, Saarialho-Kere UK. Urokinase plasminogen activator is expressed by basal keratinocytes before interstitial collagenase, stromelysin-1, and laminin-5 in experimentally induced dermatitis herpetiformis lesions. J Invest Dermatol. 1997;108(1):7-11.

[17] Salmela MT, Pender SL, Reunala T, MacDonald T, Saarialho-Kere U. Parallel expression of macrophage metalloelastase (MMP-12) in duodenal and skin lesions of patients with dermatitis herpetiformis. Gut. 2001;48(4):496-502.

[18] Ohlsson K, Olsson I. The neutral proteases of human granulocytes. Isolation and partial characterization of two granulocyte collagenases. Eur J Biochem. 1973;36(2):473-481.

[19] Wilhelm SM, Collier IE, Marmer BL, Eisen AZ, Grant GA, Goldberg GI. SV40-transformed human lung fibroblasts secrete a $92-\mathrm{kDa}$ type IV collagenase which is identical to that secreted by normal human macrophages. J Biol Chem. 1989;264(29):1721317221.

[20] Xie B, Dong Z, Fidler IJ. Regulatory mechanisms for the expression of type IV collagenases/gelatinases in murine macrophages. J Immunol. 1994;152 (7):3637-3644.

[21] Salo T, Lyons JG, Rahemtulla F, Birkedal-Hansen $H$, Larjava $H$. Transforming growth factor- $\beta 1$ upregulates type IV collagenase expression in cultured human keratinocytes. J Biol Chem. 1991;266(18): 11436-11441. 
[22] Leppert D, Waubant E, Galardy R, Bunnett NW, Hauser SL. T cell gelatinases mediate basement membrane transmigration in vitro. J Immunol. 1995;154(9):4379-4389.

[23] Bullen EC, Longaker MT, Updike DL, et al. Tissue inhibitor of metalloproteinases-1 is decreased and activated gelatinases are increased in chronic wounds. J Invest Dermatol. 1995;104(2):236-240.

[24] Okada Y, Katsuda S, Okada Y, Nakanishi I. An elastinolytic enzyme detected in the culture medium of human arterial smooth muscle cells. Cell Biol Int. 1993;17(9):863-869.

[25] Paquet P, Nusgens BV, Pierard GE, Lapiere CM. Gelatinases in drug-induced toxic epidermal necrolysis. Eur J Clin Invest. 1998;28(7):528-532.

[26] Stähle-Bäckdahl M, Inoue M, Guidice GJ, Parks WC. $92-\mathrm{kD}$ gelatinase is produced by eosinophils at the site of blister formation in bullous pemphigoid and cleaves the extracellular domain of recombinant $180-\mathrm{kD}$ bullous pemphigoid autoantigen. J Clin Invest. 1994;93(5):2022-2030.

[27] Janoff A, Scherer J. Mediators of inflammation in leukocyte lysosomes. IX. Elastinolytic activity in granules of human polymorphonuclear leukocytes. J Exp Med. 1968;128(5):1137-1155.

[28] Turto H, Lindy S, Uitto VJ, Wegelius O, Uitto J. Human leukocyte collagenase: characterization of enzyme kinetics by a new method. Anal Biochem. 1977;83(2):557-569.

[29] Postlethwaite AE, Lachman LB, Mainardi CL, Kang $\mathrm{AH}$. Interleukin 1 stimulation of collagenase production by cultured fibroblasts. J Exp Med. 1983;157 (2):801-806.

[30] Murphy G, Reynolds JJ, Werb Z. Biosynthesis of tissue inhibitor of metalloproteinases by human fibroblast in culture. Stimulization by $12-\mathrm{O}-$ tertraphorbol13-acetate and interleukin-1 in parallel with collagenase. J Biol Chem. 1983;260:3062-3066.

[31] Clark SD, Wilhelm SM, Stricklin GP, Welgus HG. Coregulation of collagenase and collagenase inhibitor production by phorbol myristate acetate in human skin fibroblasts. Arch Biochem Biophys. 1985;241(1):36-44.

[32] Welgus HG, Jeffrey JJ, Eisen AZ, Roswit WT, Stricklin GP. Human skin fibroblast collagenase: interaction with substrate and inhibitor. Coll Relat Res. 1985;5(2):167-179.

[33] Sellers A, Murphy G, Meikle MC, Reynolds JJ. Rabbit bone collagenase inhibitor blocks the activity of other neutral metalloproteinases. Biochem Biophys Res Commun. 1979;87(2):581-587.

[34] Liu Z, Shipley JM, Vu TH, et al. Gelatinase Bdeficient mice are resistant to experimental bullous pemphigoid. J Exp Med. 1998;188(3):475-482. 


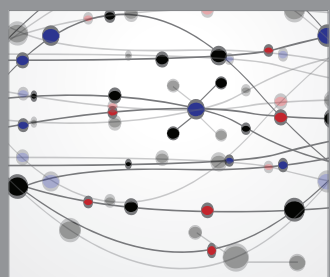

The Scientific World Journal
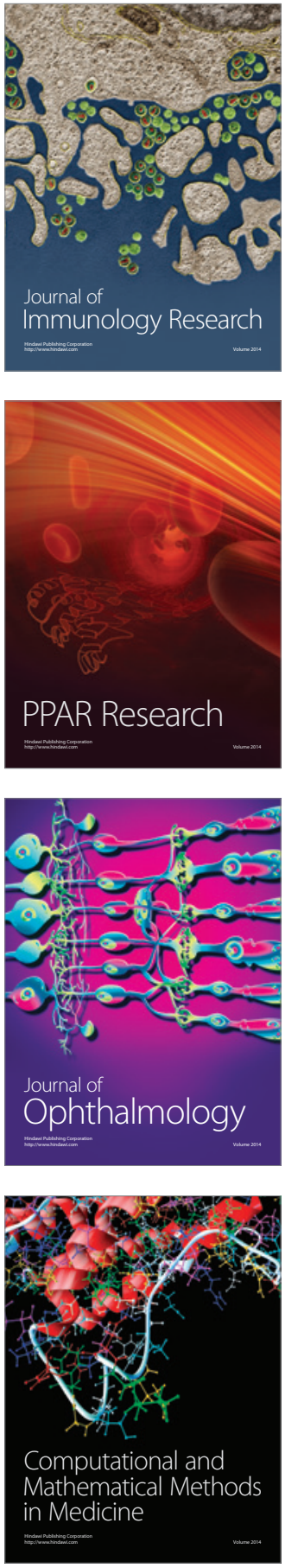

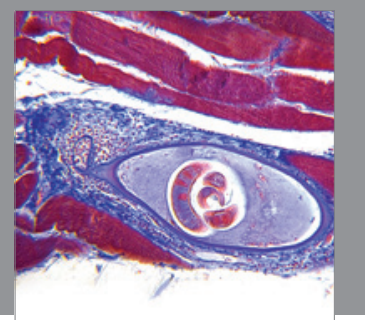

Gastroenterology

Research and Practice
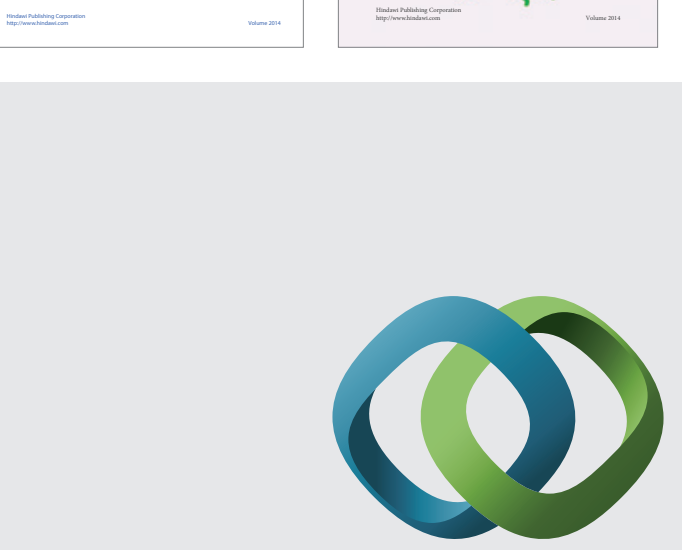

\section{Hindawi}

Submit your manuscripts at

http://www.hindawi.com
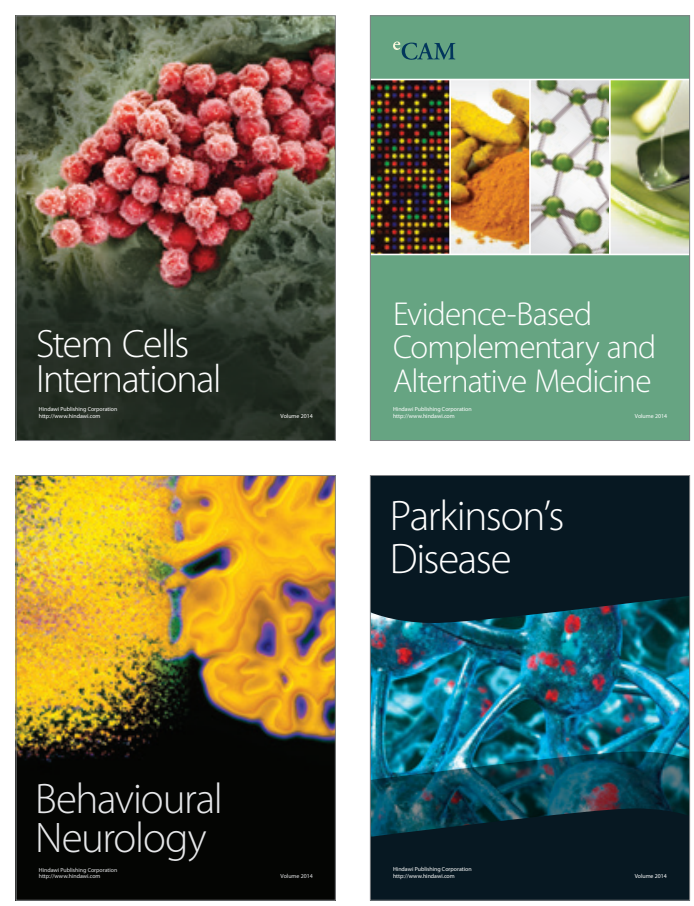

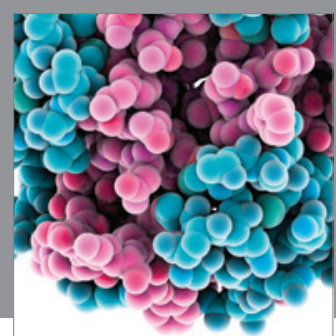

Journal of
Diabetes Research

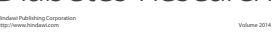

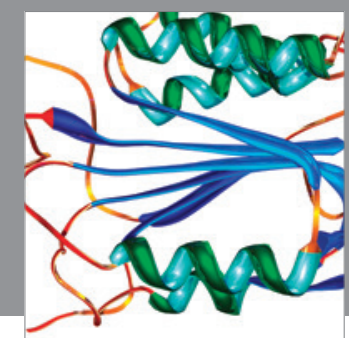

Disease Markers
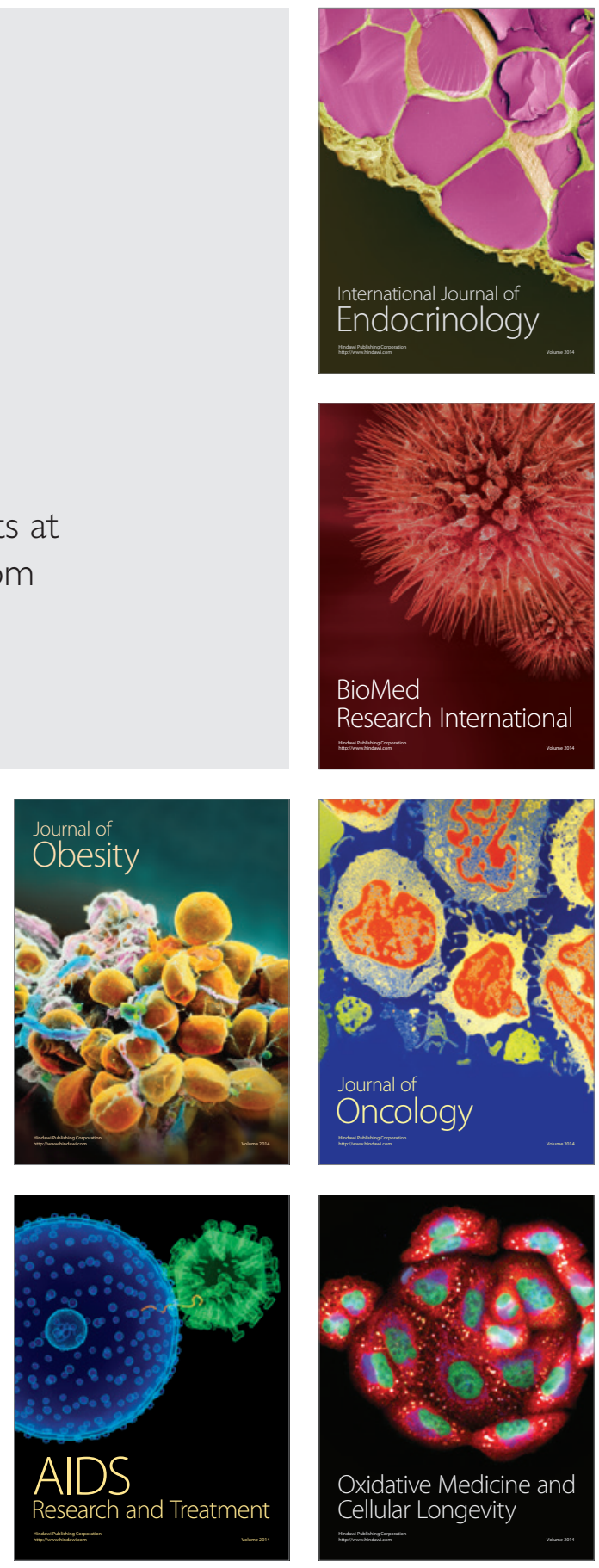